\title{
Multimedia: Where are we now and where do we go from here?
}

\author{
JAMES M. KIELEY \\ Miyazaki International College, Miyazaki, Japan
}

\begin{abstract}
This paper comments on the current state and future role of computer-based multimedia in psychology instruction. It includes practical tips on getting started with successful multimedia production. It also introduces a new World-Wide Web site archiving this information as well as other useful multimedia production and teaching resources.
\end{abstract}

There is empirical evidence to support the claim that properly constructed multimedia materials can be an aid in many instructional situations (Large, 1994). This paper will discuss how the deployment of digital multimedia in the psychology classroom has been impeded in the past and present solutions that may serve as a road map for aspiring multimedia producers in the future.

Currently, there is no adequate information describing how, and the extent to which, multimedia is being used by teaching psychologists. This is unfortunate. Multimediabased instruction has surely had successes and failures. Successes are usually only known by the limited number of students who have sat through a given class and perhaps by a fortunate few colleagues who have seen an innovative demonstration presented at a conference or who have attended a workshop.

Word of failed attempts at fully exploiting the capabilities of multimedia may be more familiar. Specific examples of substandard efforts are rarely identified or analyzed in detail. Too often people who have had bad experiences either in attempting to produce their own multimedia presentation or in viewing a piece produced by someone else entirely dismiss the potential instructional benefits. Multimedia, like any other teaching tool, can be misapplied as filler activity that shifts focus from important course content. However, it can also be properly used and provide students with a rich form of information input they could not otherwise experience.

\section{What Do We Mean by Multimedia?}

A literature search provides an interesting perspective from which to view the use of multimedia by psychologists. Among materials included in a 1975 index for psychology-related teaching materials produced by the National Information Center for Educational Media were "films, videotapes, transparencies, recordings and multimedia presentations." In effect, the term multimedia used at this time equated to educational materials that were

Correspondence should be addressed to J. M. Kieley, The Claremont Graduate School, Academic Computing Building, 130 E. 9th St., Claremont, CA 91711 (e-mail: jim@,cgs.edu). audiovisual in nature. There were no personal computers, CD-ROMs, or laserdiscs.

Recent publications apply the term much differently. Multimedia is used as a high-tech buzzword. Films, videotapes, transparencies, and audio tape recordings are often inferred to be inferior information delivery technologies because they are not computer-based and are less interactive. The popular trend is to reserve the multimedia moniker for information accessed from a computer, although the audio and video presented on a computer are often of inferior quality to the older stand-alone technologies. Certainly, the concept of interactive multimedia that permits asynchronous user control of a presentation sequence is rightfully reserved for the digital information age. On the other hand, the term multimedia alone simply means the simultaneous presentation of more than one form of media, be it text, audio, standard graphics, 3-D graphics, animation, or other video in any combination.

\section{Where Did We Come From?}

The first generation of computer-based multimedia applications used by psychologists is composed of programs written in standard high-level languages, such as Basic, Pascal, and $\mathrm{C}$, that present various combinations of text, simple graphics, and, perhaps, sounds.

Development using this approach tended to be very labor intensive; thus, very little of it took place. In some cases, individuals or departments supporting some of the more ambitious undertakings attempted to offset development costs by licensing the finished product to educational distributors or selling licensed copies directly. In most cases, these products were usable only as distributed. They could not be modified in order to fit a custom application at a given site. During this period spanning much of the 1980s, the Internet was less developed than it is today, which made product distribution costly and more difficult. Thus, a number of factors negatively impacted on the production, distribution, and sharing of computerbased multimedia materials among psychologists.

\section{Where Are We Now?}

The second generation of computer-based multimedia is composed of presentations assembled with the assis- 
tance of special authoring tools. Authoring programs are used for creating and assembling computer-assisted learning (CAL) and other educational software packages. These programs typically provide a menu-driven, mousecontrolled, graphical user interface that can be used to organize and control a presentation. The packages vary in their interfaces, commands, features, and versatility. Product information for over 80 different authoring programs can be accessed at a World-Wide Web (WWW) site (United States Government, 1995).

The early versions of popular multimedia authoring programs were typically limited to presenting text and graphics on a stand-alone computer, but they generally did offer a standard look and a degree of user-controllable interactivity in the finished product. More recently, many authoring packages have added support for audio, animation, and limited video. The more feature-rich packages today also include powerful scripting languages, the ability to control external devices such as laserdisc players, and the ability to output a finished product to videotape.

Many current versions of authoring programs provide some support for importing/exporting program componentș, Some multiplatform products permit a presentation produced on one computer platform (e.g., a Macintosh) to be played on another (e.g., Windows). Although even a limited capability to share data between packages is desirable, the great number of continuing incompatibilities between authoring programs negatively affects the widespread adoption of computer-based multimedia for instructional use.

Some small schools and departments cannot afford the same high-end authoring packages that others can provide. The bells and whistles found in the more expensive software generally are not supported in more affordable programs. High-end packages also tend to require highend support, which must be either found on campus or purchased from software publishing companies at premium prices. Low-end products, on the other hand, are generally less flexible and less frequently updated.

\section{Where Do We Go From Here?}

The third generation of computer-based multimedia is just beginning, and it can be said to be defined by two principles: (1) component elements making up a given presentation consist of individual objects ${ }^{1}$ that can be shared across operating system and presentation player environments, and (2) presentations are network-friendly.

The first requirement introduces the platformindependent "open-systems" concept to multimedia production. It is beneficial for teaching psychologists contemplating instructional use of multimedia because it specifies that the component pieces making up a particular presentation can be shared independently of the particular computer type and authoring program being used.

The time taken to collect and digitize (or produce from scratch) the sounds, graphics, or videos necessary to demonstrate a particular psychological phenomenon can be significant. This dissuades many from attempting to produce what may turn out to be very effective classroom presentations. Viewed in isolation, the return on a multimedia production may seem low. For example, assume it takes $3 \mathrm{~h}$ to prepare a demonstration of a perceptual illusion that will last only $5 \mathrm{~min}$ during class. If a particular course is taught twice a year, it would take nearly 20 years before the class demonstration time equalled the original preparation time. The fact that a given multimedia presentation may be shown only once or on a few occasions before the content or the underlying technology becomes dated is another factor discouraging multimedia production. The possibility that several instructors scattered across the world may be preparing very similar demonstrations further underscores the problem. The price of any redundancy is other work that will go undone.

The solution to this dilemma is to promote as much free sharing of digitized psychological demonstrations as is possible. Sharing should extend beyond simply permitting public access to a finished product; it should also include open access to the objects that make up a given presentation. Only with this level of access will the psychology community be able to avoid unnecessary redundant effort and, at the same time, be able to tailor a given presentation to the specific needs of a particular class.

This solution willsequire the appropriate support technologies. Not only will open-systems compliant programs need to be used to produce shareable components, but the library of objects will need to be easily and inexpensively accessible. The obvious way for the latter requirement to be met is via the Internet. The specific set of network protocols and programs necessary to support this sharing model will vary as a function of the specific instructional application.

Some forms of real-time multimedia sharing are possible with existing networks and technologies; others are not. Real-time sharing means that a presentation stored on a server can be played back directly from other machines located on the network at an acceptable quality level. In contrast to real-time sharing, delayed sharing of almost any digitized component is generally possible with modern academic networks. This means that it is feasible for individuals located at great distances from one another to upload and download objects and presentations from multimedia archives. Once a particular piece has been transferred to a destination machine, it can be played back or edited.

The primary technical factor constraining the suitability of local area network (LAN) or wide area network (WAN) technology for a given multimedia application is available bandwidth. Available bandwidth varies as a function of the particular form of media being transmitted, the size and duration of the presentation components, the types of compression used, whether or not a synchronous presentation being transmitted to multiple workstations is being unicast or multicast, the amount of competing network traffic, and the minimum network speed (i.e., bottleneck speed) at any point between the sending and receiving computers.

Text, smaller graphics, lower quality audio and video, good compression schemes, multicasting (a protocol that 
allows multiple workstations to simultaneously share the same transmission), and homogeneous networks with limited numbers of machines that are dedicated to a specific use are bandwidth-friendly factors. Large graphics, lengthy and higher quality audio and video, poor compression schemes, unicasting (any protocol that requires multiple workstations to receive separate identical transmissions), and large busy heterogeneous networks are factors that limit available bandwidth.

\section{The World-Wide Web Model and Instructional Multimedia}

The WWW provides a compelling model for the future direction of multimedia teaching applications. The WWW was originally designed as a presentation system to support hypertext-based linking between noncontiguous text located on computer networks. It also provided a limited ability to present other forms of media including graphics, sound, and video. Subsequent development efforts have resulted in gateway support for a wide range of additional information resources that can be accessed from a common interface (see Kieley, 1996).

Today, all multimedia development should be headed in this direction. That is, regardless of the particular authoring tool used to create a presentation component, wherever possible multimedia materials should be exportable in a format that can be accessed from a WWWcompatible browser. This will provide the technological basis necessary for psychologists to immediately share a wide assortment of multimedia-based instructional materials. In situations where network bandwidth limitations would make real-time access impractical, a presentation and its associated components should be retrievable via FTP (see Kieley, 1993, for additional information about FTP).

Although some types of multimedia presentations may be unsuitable for current networks, computers, and software, the situation will soon change. At NCSA, where much of the previous important WWW development has taken place, researchers are currently working on a new technology called VMosaic. VMosaic permits fast workstations operating on fast networks to access and display highquality video. Soon, this or similar technologies will be widely available.

The long-term future of multimedia in psychology instruction is no different from what it will be anywhere else, be it within an academic discipline or elsewhere. We will soon see faster and more reliable networks and a merging of technology-based media delivery systems. The network service that supports a telephone will be no different from the service that supports television or information that is exchanged between computers. This could all be fully realized in another decade.

\section{A Call for Shared Multimedia Development Among Psychologists}

Technological issues aside, it can be argued that the largest impediment to the widespread use of multimedia in the psychology classroom is that most of the develop- ment activity to date has been carried out in isolation. That is, a largely unknown list of individuals working at an unknown number of colleges and universities have used multimedia in an instructional context, but it is hard to know what actually has been done and how. There are very few documented uses of computing-based multimedia as a psychology teaching tool. This suggests that a successful implementation of multimedia at a given school in a particular department is too often a hit-or-miss proposition based on the knowledge, initiative, and tools harbored by a limited number of individuals.

Although in theory it may be possible for interdepartmental multimedia technical resource sharing, differences across disciplines frequently discourage this. Thus, while the sharing of expertise within a campus is desirable, a more promising and yet largely untapped resource is the expertise that is available across campuses. Small colleges frequently have few, if any, multimedia experts. Furthermore, even individuals working at large campuses may find that it literally takes weeks to obtain technical help with a presentation under development.

For years, USENET newsgroups have provided technical computing people with free access to an international group of experts on a range of technical subjects, including multimedia development. Publicly accessible newsgroups are also supplemented with private mailing lists focusing on more specific application issues.

Sharing of technical expertise is certainly desirable, but an even greater benefit for teaching psychologists interested in incorporating multimedia into their instruction could come from pooling source materials. It is a waste of time for several instructors needing similar materials for class demonstrations to have to duplicate search and production efforts. It would make more sense if a central index of previously digitized materials could be searched from a single site. For this suggestion to be effective, there will, of course, have to be a commitment among faculty members located at different campuses to share their multimedia products.

To help promote this type of sharing, a WWW server has been established at http://acb2.cgs.edu/multi.html that includes links to a variety of multimedia information resources (see also Table 1). At this same site is information on locations where multimedia materials produced by psychologists can be uploaded and downloaded. Also available are additional tips on how to get started in multimedia production and a searchable repository of postings from the USENET newsgroup comp.multimedia.

\section{Getting Started}

Keeping up with the pace of changes in the field of information technology is more than a full-time occupation for anyone; therefore, it is helpful to have a few practical tips that will serve as a navigation tool in identifying what to learn about classroom multimedia deployment and production.

Faculty tend to fall into one of three groups when it comes to incorporating multimedia and other new technologies into their teaching: (1) those who don't want to 
Table 1

Internet-Accessible Multimedia Information Sites

\begin{tabular}{|c|c|}
\hline Location & Description \\
\hline \multicolumn{2}{|c|}{ General Purpose } \\
\hline $\begin{array}{l}\text { http://www.info.apple.com/dev/ } \\
\text { http://www.mcli.dist.maricopa.edu/authoring/ } \\
\text { http://viswiz.gmd.de/MultimediaInfo } \\
\text { http://morse.colorado.edu/ armstros/playhouse.html } \\
\text { http://www.lib.siu.edu/mmwwwpc/mmwwwpc.html } \\
\text { http://netlab.itd.nrl.navy.mil/MM.html } \\
\text { http://found.cs.nyu.edu/CAThome_new.html } \\
\text { http://www.acm.uiuc.edu/rml/ } \\
\text { http://www.acs.oakland.edu/oak/SimTel/win3/multimed.html } \\
\text { http://www.cen.com/mw3/ }\end{array}$ & $\begin{array}{l}\text { EMASHE } \\
\text { Multimedia Authoring Page } \\
\text { MultimediaInfo Index } \\
\text { Multimedia Playhouse } \\
\text { Multimedia WWW PC } \\
\text { Navy Multimedia Page } \\
\text { NYU Center for Digital Multimedia } \\
\text { Rob's Multimedia Lab } \\
\text { Simtel Multimedia Directory for Windows } \\
\text { X-Windows based Multimedia }\end{array}$ \\
\hline \multicolumn{2}{|c|}{ Commercial Products and Companies } \\
\hline $\begin{array}{l}\text { http://www.lib.siu.edu/tbkwww/tbkwww.html } \\
\text { http://www.primenet.com/ laig/dcweb/index.html } \\
\text { http://the-tech.mit.edu/KPT/KPT.html } \\
\text { http://www.mcli.dist.maricopa.edu/director/ } \\
\text { http://www.mae.com/ } \\
\text { http://sunsite.sut.ac.jp/multimed/ } \\
\text { http://www.voyager.com }\end{array}$ & $\begin{array}{l}\text { Asymetrix Toolbook } \\
\text { Digital Currency } \\
\text { Kai's Place } \\
\text { Macromedia Director } \\
\text { MAEstro (for UNIX) } \\
\text { Sunsite Multimedia } \\
\text { Voyager }\end{array}$ \\
\hline \multicolumn{2}{|c|}{ Movies and Animation } \\
\hline $\begin{array}{l}\text { http://quicktime.apple.com } \\
\text { http://www.acs.oakland.edu/oak/simtelnet/msdos/animate } \\
\text { http://www.rmplc.co.uk./eduweb/sites/terrymen/moviel.html } \\
\text { http://www2.cinenet.net/GWEB/links.html } \\
\text { http://www.arc.umn.edu/GVL/Software/mpeg.html } \\
\text { http://www.crs4.it/HTML/LUIGI/MPEG/mpegfaq.html } \\
\text { http://www.eit.com/techinfo/mpeg/mpeg.html } \\
\text { http://www.netvideo.com/ } \\
\text { http://www.onshore.com/quicktime/ }\end{array}$ & $\begin{array}{l}\text { Apple's Quicktime On-line } \\
\text { DOS Animation Utils } \\
\text { FineCut Moviemaking } \\
\text { G-Web links } \\
\text { How to Make MPEG Movies (UNIX only) } \\
\text { MPEG FAQ } \\
\text { MPEG Technical Info } \\
\text { Netvideo } \\
\text { Quicktime FAQ }\end{array}$ \\
\hline \multicolumn{2}{|c|}{ Sound and Music } \\
\hline $\begin{array}{l}\text { http://datura.cerl.uiuc.edu/ } \\
\text { http://www.eeb.ele.tue.nl/midi/index.html } \\
\text { http://wwwtios.cs.utwente.nl/say/form/ }\end{array}$ & $\begin{array}{l}\text { CERL Sound Group } \\
\text { MIDI and Music Resources } \\
\text { Text to Sound }\end{array}$ \\
\hline \multicolumn{2}{|c|}{$3-D$} \\
\hline $\begin{array}{l}\text { http://www.cnam.fr/fractals/anim.html } \\
\text { http://www.lightside.com: } 80 / \sim \text { dani/ } \\
\text { http://vrml.wired.com }\end{array}$ & $\begin{array}{l}\text { Fractals } \\
\text { 3D Web } \\
\text { VRML }\end{array}$ \\
\hline
\end{tabular}

get left behind and who are willing to invest the time necessary to examine the majority of new innovations, (2) those who dismiss all such pursuits as a misdirected waste of time, and (3) those who fall somewhere in between. On balance, Approach 3 may be most reasonable. It can be approximated by pursuing the following tenets:

1. Remain open to the possibility of enhancing instruction through multimedia.

2. Stay abreast of major technological developments used by fellow educators.

3. When undertaking multimedia production, focus on obtaining closure on each project; scale the ambitiousness of a project to both ability levels and available time.

4. Pursue the "lowest tech" approach available to meet the majority of multimedia goals (development environments with fewer bells and whistles generally carry the least amount of technical overhead; low-tech approaches also are often easier to share across platforms).
5. Ensure that the available hardware and software are sufficient for the project.

6. Ensure that the appropriate level of technical support necessary to complete a project is available.

7. Publicize efforts and freely share products and technological expertise.

Once the above principles are understood, the next step is having some idea of how to apply them. ${ }^{2}$ A first step would be to survey departments and nearby colleagues on how they are currently using multimedia in instruction. Any information they can provide should be helpful; however, don't be surprised if you end up feeling like you are on your own at this stage.

A second step, assuming a school or department has a Web server, would be to provide some type of on-line class information to students. Properly taught, WWW publishing is easy to learn. Even if a first effort is limited to a text-only syllabus, such an exercise will give 
an instructor a sense of accomplishment and competence. The addition of graphics and other media can quickly follow an initial success and provide a better sense of what network-based multimedia publishing is all about.

A third step would be to produce a simple WWWaccessible instructional module. This could consist of a graphics presentation shown in class that resembles a traditional slide show. A fourth step would be to extend this third step to include a wider number of classroom presentations, substituting WWW-accessible materials for class handouts, setting up simple Web-based experiments in which students can participate (e.g., Krantz, 1995), and making short audio-video segments accessible from network workstations.

Each of these suggested applications can be implemented without relying on an authoring program. Each application is network-based and thus potentially shareable both locally and over the Internet.

The typical HTML editing tools that are used for assembling WWW documents are not sufficient to construct animations or to edit sound and videos. Furthermore, the current speed of the majority of computing networks is not sufficient to support full-screen video with highquality sound. Therefore, authoring programs may still have their place. It is recommended that individuals interested in using authoring programs select products that are network-friendly and that support a wide range of export options. This will maximize shareability of any multimedia product.

\section{Conclusions}

It can be argued that three things will have to occur if the use of computer-based multimedia materials is going to approach its potential in psychology instruction: (1) a movement toward open-systems-type authoring environments that produce component materials that can be used on a variety of computer platforms, (2) intercampus sharing among psychologists of previously prepared noncopyrighted component materials (e.g., graphics, sounds, and video) as well as integrated presentations, and (3) a further evolution of computer networks.

Properly selected and administered multimedia demonstrations at a minimum embellish the traditional psychology classroom. In some instances, students can have classroom experiences that otherwise would be totally unobtainable. Interactive multimedia learning materials that students can control on their own go one step farther: they allow students to explore answers to questions that they personally find interesting and also to pursue learning at their own pace.

The WWW model of easy authoring and information sharing has been responsible for its fantastic growth. Teaching psychologists should borrow from this model. Due to fiscal constraints, crowded classrooms, and the evolving nature of advanced learning, in the near future the ideas of an open campus and distance learning will become more commonplace. This eventuality will bring with it a greater demand for information-rich, remotely accessible, interactive multimedia learning resources. The Internet will play an important role in addressing this requirement.

\section{REFERENCES}

KIELEY, J. M. (1993). Integrating remotely accessible information services into psychology instruction and research. Behavior Research Methods, Instruments, \& Computers, 25, 287-294.

KIELEY, J. M. (1996). CGI scripts: Gateways to World-Wide Web Power. Behavior Research Methods, Instruments, \& Computers, 28, 165-169.

Krantz, J. H. (1995). Psychology experiments on the Net [On-line]. Available URL: http://psych.hanover.edu/APS/exponnet.html

LARGE, A. (1994). Multimedia and comprehension: A cognitive study, Journal of the American Society for Information Science, 45, 515-528.

National Information Center for Educational MEdia. (1975). Index to psychology (multimedia). Los Angeles: University of Southern California.

United States Government. (1995). AuthorBase [On-line]. Available URL: http://wwwetb.nlm.nih.gov/authorb/index.html

\section{NOTES}

1. An object can consist of a single sound or graphic, or it can consist of a group of related media that work together in a presentation sequence. For example, a single object might comprise a video section with a separately recorded and later synchronized sound track.

2. Third-generation multimedia deployment and development will be severely restricted within academic environments that do not provide full Internet connectivity and basic Internet services, such as e-mail, USENET news, FTP, and the WWW.

(Manuscript received November 13, 1995; revision accepted for publication February 12, 1996.) 